\title{
Los estudios de fecundidad en poblaciones contemporáneas como referentes directos para los estudios paleodemográficos*
}

\author{
Patricia Olga Hernández Espinoza** \\ Lourdes Márquez Morfín***
}

Las limitantes del trabajo paleodemográfico, como la obtención de datos sobre la fecundidad de las sociedades antiguas, han impulsado el desarrollo de estrategias metodológicas para obtener esta información con algún grado de certeza. En este estudio contrastamos dos tipos de metodologías para obtener datos sobre fecundidad: la paleodemográfica basada en el análisis bioarqueológico de una serie esquelética maya prehispánica, utilizando el modelo de Weiss, y la metodología demográfica moderna aplicada a datos censales de Quintana Roo, con los que se calcularon varios indicadores de fecundidad. Los resultados muestran que el método estadístico que hemos aplicado en nuestras investigaciones permite tener un acercamiento confiable a los niveles de fecundidad y mortalidad que pudieron haber tenido estos grupos en el pasado, pues las estimaciones paleodemográficas presentan valores consistentes con la información obtenida de los censos, con un cálculo de cuatro a seis hijos para el grupo maya prehispánico de Chac Mool.

Palabras clave: paleodemografía, fecundidad, Quintana Roo, mayas. Fecha de recepción: 22 de octubre de 2012.

Fecha de aceptación: 17 de mayo de 2013.

\section{Fertility Studies in Contemporary Populations as Direct References for Paleodemographic Studies}

The constraints on paleodemographic work, such as collecting data on the fertility of ancient societies, have prompted the development of methodological strategies to obtain this information with some degree of accuracy. In this study, we contrast two types of methodologies to obtain data on fertility: paleodemographic, based on the bioarchaeological analysis of a pre-Hispanic Maya skeletic series, utilizing Weiss's model, and modern demographic methodology applied to census data from Quintana Roo, used to calculate various fertility indicators. The results show that the statistical method we have used in our research makes it possible to have a reliable approach to the levels of fertility

* La versión preliminar de este trabajo se presentó en la VIII Reunión de Investigación Demográfica de la Somede en 2006.

** Centro INAH Sonora. Correo electrónico:<olga_hernandez@inah.gob.mx>, <patriciaolga.hernandezespinoza@gmail.com>.

*** Escuela Nacional de Antropología e Historia. Correo electrónico: <rlmorfin@ gmail.com>.

$\overline{\text { ESTUDIOS DEMOGRÁFICOS Y URBANOS, VOL. 29, NÚM. } 2 \text { (86), 2014, 341-363 }}$ 
and mortality these groups might have had in the past, since the paleodemographic estimates have values consistent with the information obtained in the censuses, with a calculation of four to six children for the pre-Hispanic Maya group of Chac Mool.

Key words: paleodemography, fertility, Quintana Roo, Maya.

El interés por conocer los perfiles demográficos de las sociedades antiguas ha llevado a los investigadores interesados en el tema a considerar varias estrategias metodológicas, pues los datos son deficientes e incompletos (Brass, 1975). Los estudios paleodemográficos se basan en la distribución por edad a la muerte de individuos representados por sus esqueletos (series osteológicas). Se considera que son "datos eficientes e incompletos" ya que no todos los grupos de edad están representados. De los indicadores demográficos calculados a partir de estas series, los más polémicos se refieren a los niveles de fecundidad alcanzados por las sociedades en el pasado. Para obtener este tipo de información se ha utilizado el modelo teórico de Weiss (1973: 31-41), desarrollado considerando la experiencia reproductiva de 13 sociedades antropológicas, ${ }^{1}$ o aplicando las tablas de modelo de vida que este mismo autor propone (Weiss, 1973, apéndice C: 115-186), en las que se sugieren los niveles de mortalidad y fecundidad de acuerdo con la esperanza promedio de vida al nacimiento. Otros investigadores utilizan información de fecundidad derivada de la experiencia reproductiva de grupos cazadores-recolectores contemporáneos (Hill y Hurtado, 1996; Márquez y Hernández, 2001; Meindl y Russell, 1998).

Para el caso de las poblaciones prehispánicas de México se han realizado diversos estudios paleodemográficos que han utilizado series osteológicas mayas de los periodos Clásico y Posclásico de Yucatán, Chiapas, Campeche y Quintana Roo, y han aprovechado diversas fuentes de datos, como los datos arqueológicos, las fuentes etnográficas y la información recuperada vía tradición oral entre mujeres mayas, que aportan testimonios valiosos para conocer su fecundidad. Estas investigaciones parten del supuesto de una fecundidad alta, de alrededor de ocho hijos por mujer, con la cual es posible reponer a los hijos muertos en los primeros años de vida (Hernández Espinoza y Márquez Morfín, 2003, 2005, 2006a, 2006b y 2007; Hernández Espinoza, 2006) . ${ }^{2}$

${ }^{1} \mathrm{El}$ autor utiliza dicho término para resaltar las condiciones de vida de estos individuos, caracterizados por un escaso desarrollo tecnológico y la ausencia de un control deliberado de la fecundidad.

${ }^{2}$ De acuerdo con los paleodemógrafos Konigsberg y Frakenberg (2002), una alta mortalidad infantil supone altos niveles de fecundidad. 


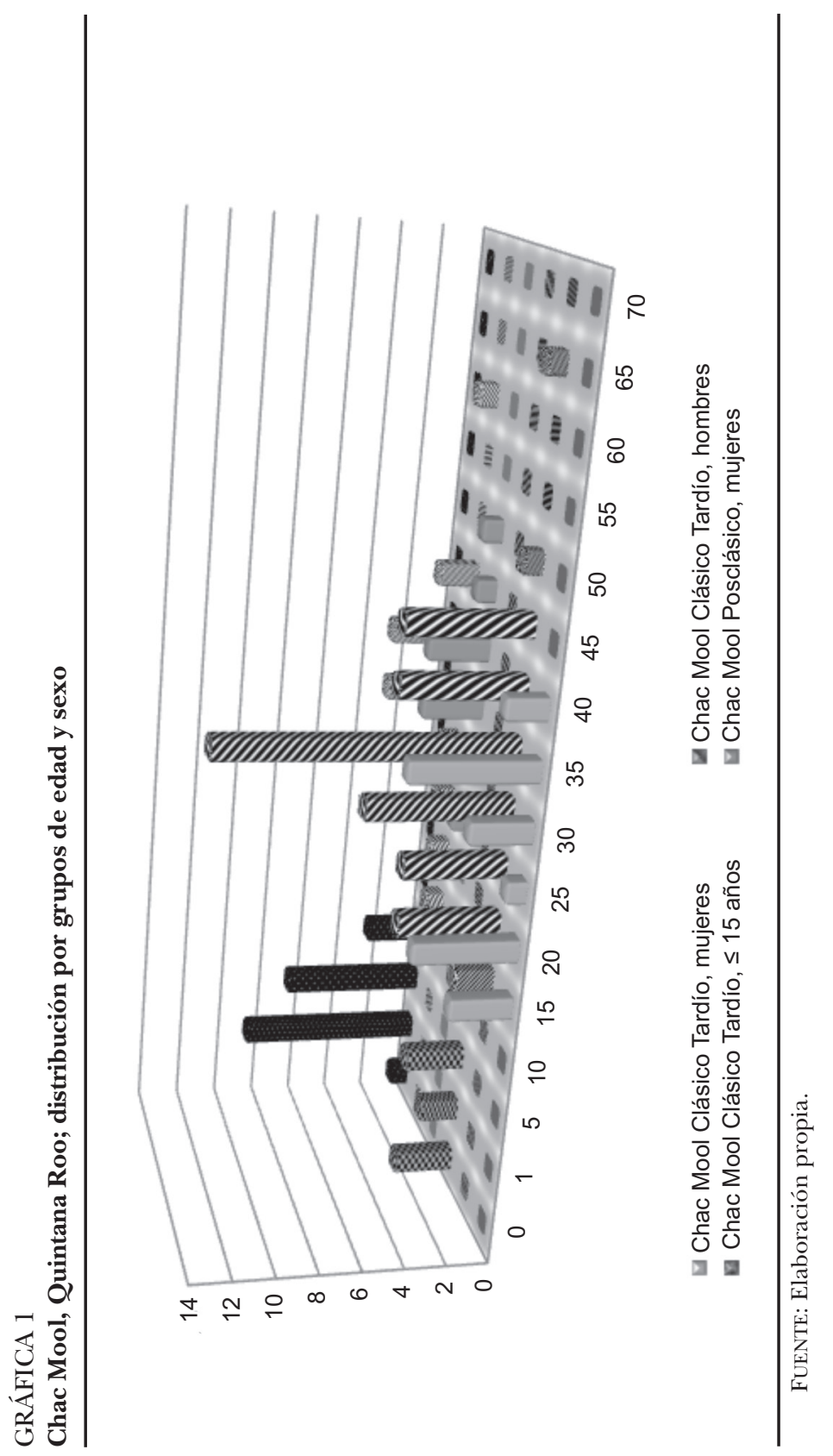


Los resultados paleodemográficos obtenidos de las series osteológicas de los sitios de Jaina, ${ }^{3}$ Xcaret y Chac Mool revelan tasas de fecundidad con niveles moderados, esto es, cuatro o cinco hijos en promedio por cada mujer que sobrevivió hasta el final de su periodo reproductivo. Consideramos que esta tasa moderada para los mayas de esos periodos se debe a una escasa sobrevivencia de las mujeres de edades mayores.

Pero si los mayas constituían una sociedad pronatalista y su régimen de fecundidad era natural, entonces, ¿por qué no suponer que habrían tenido un número más elevado de hijos?

Para responder a estos supuestos y con el fin de comprobar si los resultados obtenidos por medio de la metodología paleodemográfica son confiables, se analizó la fecundidad de las mujeres que habitan en el actual estado de Quintana Roo, examinando la información sobre fecundidad que contienen los censos de las tres últimas décadas del siglo XX. ${ }^{4}$ A partir del número de hijos vivos que cada mujer dijo haber tenido al momento del censo, se calcularon la descendencia final y las probabilidades de agrandamiento de las familias como una forma indirecta de conocer el tamaño promedio de las familias mayas contemporáneas. Posteriormente contrastamos estos resultados con los calculados para el grupo maya procedente del sitio prehispánico de Chac Mool.

Ambos métodos tienen limitantes: en el trabajo paleodemográfico, por ejemplo, la serie osteológica excavada se transforma en una cohorte ficticia para construir una tabla de mortalidad y lograr algunas series que son básicas en la aplicación del modelo de fecundidad. La principal limitante es que no siempre se recuperan todos los individuos de un sitio, y por lo tanto no todos los grupos de edad tienen una representación adecuada; otra limitante es que a veces el balance entre sexos no es proporcionado, pues conforme a las prácticas funerarias de ese tiempo, los antiguos mayas destinaban lugares de inhumación distintos para hombres, mujeres y niños según su rango social o por cuestiones rituales.

${ }^{3}$ El estudio detallado de la población de Jaina, Campeche, calcula seis hijos nacidos por mujer, de los cuales sobreviven cuatro para reemplazar a la generación de sus padres. El espacio intergenésico era de cuatro años, de los cuales tres estaban controlados por la lactancia (Hernández Espinoza y Márquez Morfín, 2007: 57-58).

${ }^{4}$ De acuerdo con los estudios antropológicos realizados en ese estado, la fecundidad inició su descenso a partir de la década de los noventa, por lo tanto las mujeres censadas en la década anterior deberían haber reportado un número elevado de hijos, pues las prácticas anticonceptivas no fueron aceptadas por las mujeres mayores de cuarenta años (Berrío Gómez, 1995; Daltabuilt Godás, 1992; Daltabuilt Godás et al., 1997; Daltabuilt Godás et al., 1994). 
En el caso de la información censal, los datos están supeditados a la declaración de las mujeres sobre el número de hijos nacidos vivos, sobrevivientes o no, por lo que los sesgos por omisión logran afectar los resultados. Las mujeres del actual estado de Quintana Roo practican la anticoncepción, sin embargo su uso ha sido paulatino y no es en forma universal, por lo que los cálculos obtenidos corresponden a los niveles moderados de fecundidad, que consideramos para las mujeres en la época prehispánica de esa zona.

\section{El sitio arqueológico de Chac Mool}

Chac Mool está situado en Punta Pájaros, entre las bahías de La Ascensión y del Espíritu Santo. Hacia el Posclásico Temprano su ubicación estratégica lo convirtió en un enclave comercial Itzáe, un punto intermedio en la ruta que seguían los mercaderes de la sal (McKillop, 2002; Stuart y Stuart, 1993). De este sitio provienen dos series osteológicas, una que corresponde al Clásico Tardío $(750-850 \mathrm{dC})$ y otra fechada para el Posclásico (1250-1519 dC).

El contexto bioarqueológico de la serie del periodo Clásico Tardío sugiere que el asentamiento tuvo las características de cualquier puerto de hoy, con una alta proporción de población masculina y un crecimiento demográfico lento (Giannisis, 2004; Hernández Espinoza y Márquez Morfín, 2006a). Resultados de investigaciones previas revelan que durante ese periodo había una distribución igualitaria de la riqueza; las mujeres tenían el mismo estatus que los hombres, y en algunos casos un poco más (González Licón y Cobos Palma, 2006; González Licón y Terrones González, 2003). El análisis de los restos óseos humanos revela problemas de salud causados por las infecciones gastrointestinales y respiratorias propias de las precarias condiciones higiénicas. La alimentación estaba basada en alimentos procesados derivados del maíz y proteínas de origen animal producto de la pesca y la caza, así como de la recolección de plantas (Márquez y Hernández, 2006).

Las investigaciones arqueológicas plantean que hubo un cambio socioeconómico notable hacia el Posclásico, cuando Chac Mool perdió su importancia como puerto comercial, para convertirse en un asentamiento pesquero más de la región. Los datos bioarqueológicos apuntan hacia cambios importantes en el patrón funerario, indicativos de diferencias sociales más marcadas; la riqueza se concentró en unos cuantos y la salud sufrió un deterioro importante. La dieta se basó 
principalmente en la explotación de recursos marinos. La población aumentó debido principalmente a un incremento en la fecundidad (Hernández Espinoza y Márquez Morfín, 2006a). Los cambios sociales, económicos y políticos, detectados por medio de los indicadores arqueológicos, influyeron en los niveles de mortalidad y fecundidad entre uno y otro periodos, por lo que la muestra se dividió metodológicamente en dos series con tasas de crecimiento distintas.

\section{Metodología paleodemográfica para estimar los niveles de fecundidad en las poblaciones antiguas}

En la paleodemografía, como ya mencionamos, los indicadores de fecundidad se obtienen a partir del modelo teórico de Weiss (1973: 31-41), desarrollado por el autor considerando la experiencia reproductiva de 13 sociedades antropológicas. ${ }^{5} \mathrm{El}$ autor tomó las tasas específicas de fecundidad o tasas por edad para obtener siete constantes que resumen los valores de las tasas específicas registradas en las poblaciones antropológicas (véase el cuadro 1). Estas constantes son la base para calcular los principales indicadores de fecundidad, lo que implica asumir que el comportamiento reproductivo de los grupos prehispánicos era similar al de estas sociedades.

Se consideran siete grupos quinquenales de edad, que comprenden la duración del periodo reproductivo femenino, tomando como inicio el grupo de 15 a 19 años y el de 45 a 49 años como el término del mismo. La forma de la curva construida con los valores de las constantes de Weiss corresponde a lo que los demógrafos llaman "una curva de fecundidad temprana" (Mier y Terán, 1998; Mier y Terán y Rabell, 1993), donde el punto máximo está ubicado en la edad K20 (grupo de 20-24 años), lo que significa que la mayoría de los hijos de los grupos estudiados se concibió entre esas edades.

El modelo utiliza la serie $L_{(x)}$ de la tabla de mortalidad, construida a partir de la distribución de edades a la muerte. Cada $L_{(x)}$ se divide entre el radix para obtener el indicador $L_{(x)}$, que representa el número de años-persona vividos que podrían haber aportado las mujeres de cada grupo de edad. A partir de este punto el modelo trabaja sólo con los siete grupos de edad referidos. Los valores de las constantes se

${ }^{5} \mathrm{El}$ autor utiliza dicho término para resaltar las condiciones de vida de estos individuos, caracterizados por un escaso desarrollo tecnológico y la ausencia de un control deliberado de la fecundidad. 


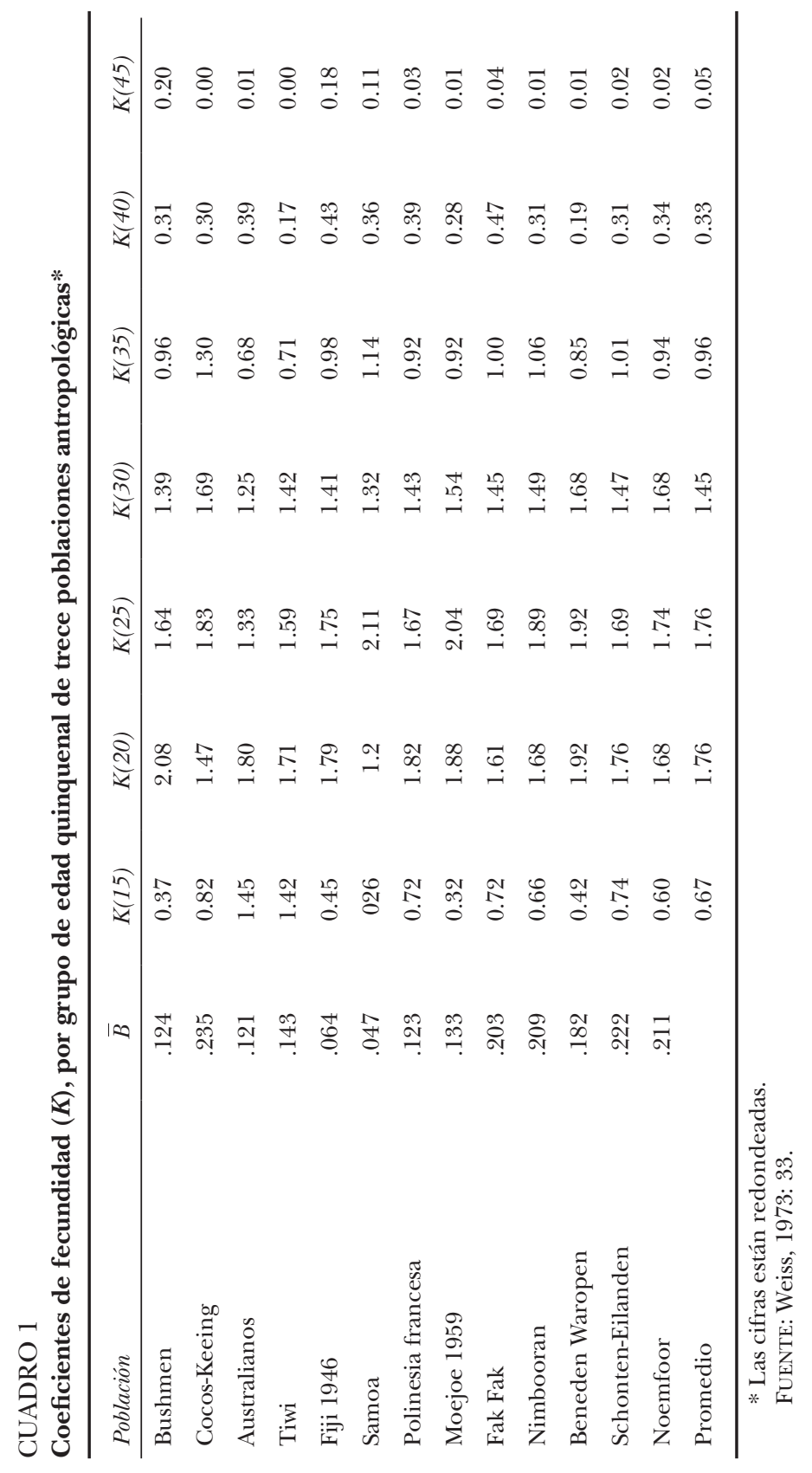




\section{GRÁFICA 2}

Curva de fecundidad de acuerdo con Weiss, 1973

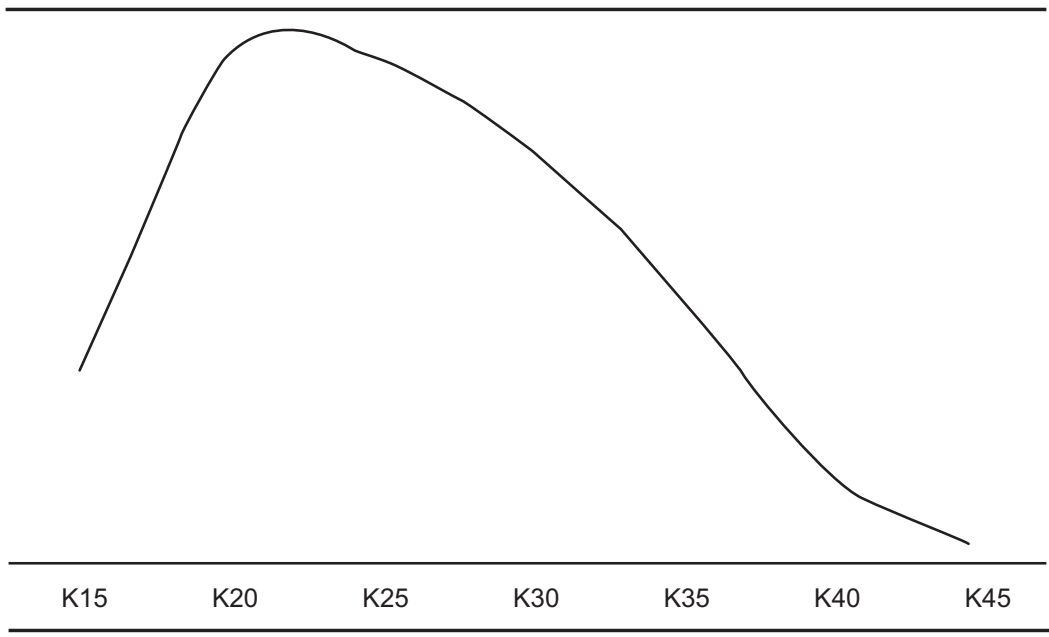

FUENTE: Elaboración propia a partir de Weiss, 1973.

multiplican por cada $L_{(x)}$, el recíproco de la sumatoria de los siete valores así obtenidos da el valor de $\beta$, que constituye el nivel general de fecundidad a partir de la relación:

$$
\beta=\frac{1}{\sum L_{(x)} K_{(x)}}
$$

Este factor se multiplica a su vez por cada constante para obtener las $F B_{(x)}$ o tasas específicas de fecundidad; la sumatoria de estos resultados es una tasa de fecundidad que significa la proporción de hijas nacidas por mujer cada año.

El resultado se multiplica por cinco (porque estamos trabajando con grupos de edad de cinco años) para obtener el número de hijas por cada mujer que sobrevivió al final de su vida reproductiva (tasa bruta de reproducción, $T B R$ ), es decir hasta la menopausia. De la multiplicación de este indicador por dos (para obtener el número de hijos e hijas) resulta el tamaño de la familia completa o descendencia final por mujer sobreviviente a los 50 años (tasa global de fecundidad o TGF).

$\mathrm{Al}$ hacer intervenir a la mortalidad podemos obtener la tasa neta de reproducción $\left(R_{0}\right)$, es decir los niveles de reproducción tomando 
en consideración la mortalidad de las mujeres, y otro indicador llamado "tamaño promedio de familia" $(F)$, que es el número promedio de hijos e hijas de una mujer que sobrevivió para reemplazar a su generación antes de llegar a la menopausia. Las relaciones matemáticas son las siguientes:
1) $F B_{(x)}=\beta K_{(x)}$
2) $T B R=\sum_{15}^{45} F B_{(x)}(5)$
3) $T G F=T B R(2)$
4) $R_{0}=\sum_{\omega}^{x} L(x) \beta(x)$
5) $F=2 R_{0} / l_{15}$

\section{Los resultados para las dos series de Chac Mool}

En el caso de la serie prehispánica de Chac Mool del Clásico Tardío se aplicó una tasa de crecimiento de 0.010 debido al lento crecimiento poblacional evidenciado por los datos arqueológicos. Para la serie del Posclásico se empleó una tasa de 0.015 . La selección de una tasa mayor de crecimiento obedece a los resultados del estudio arqueológico, que estimaron un aumento en el tamaño del asentamiento y un incremento en la densidad demográfica del sitio (Terrones González, 1998). Los resultados de los indicadores de fecundidad calculados para ambas series se presentan en la gráfica 3.

\section{Probabilidades de crecimiento de las familias a partir de la información censal}

La demografía moderna se vale de varias técnicas para obtener información sobre los niveles de fecundidad de una población. La mejor forma de obtenerla es con base en las encuestas de fecundidad, pero también es posible conseguirla de la información censal a partir de una pregunta que se plantea a las mujeres censadas: ¿Cuántos hijos ha tenido usted? Las respuestas se clasifican de acuerdo con la edad de la mujer y el número que se anota es el total de hijos que declaró haber 


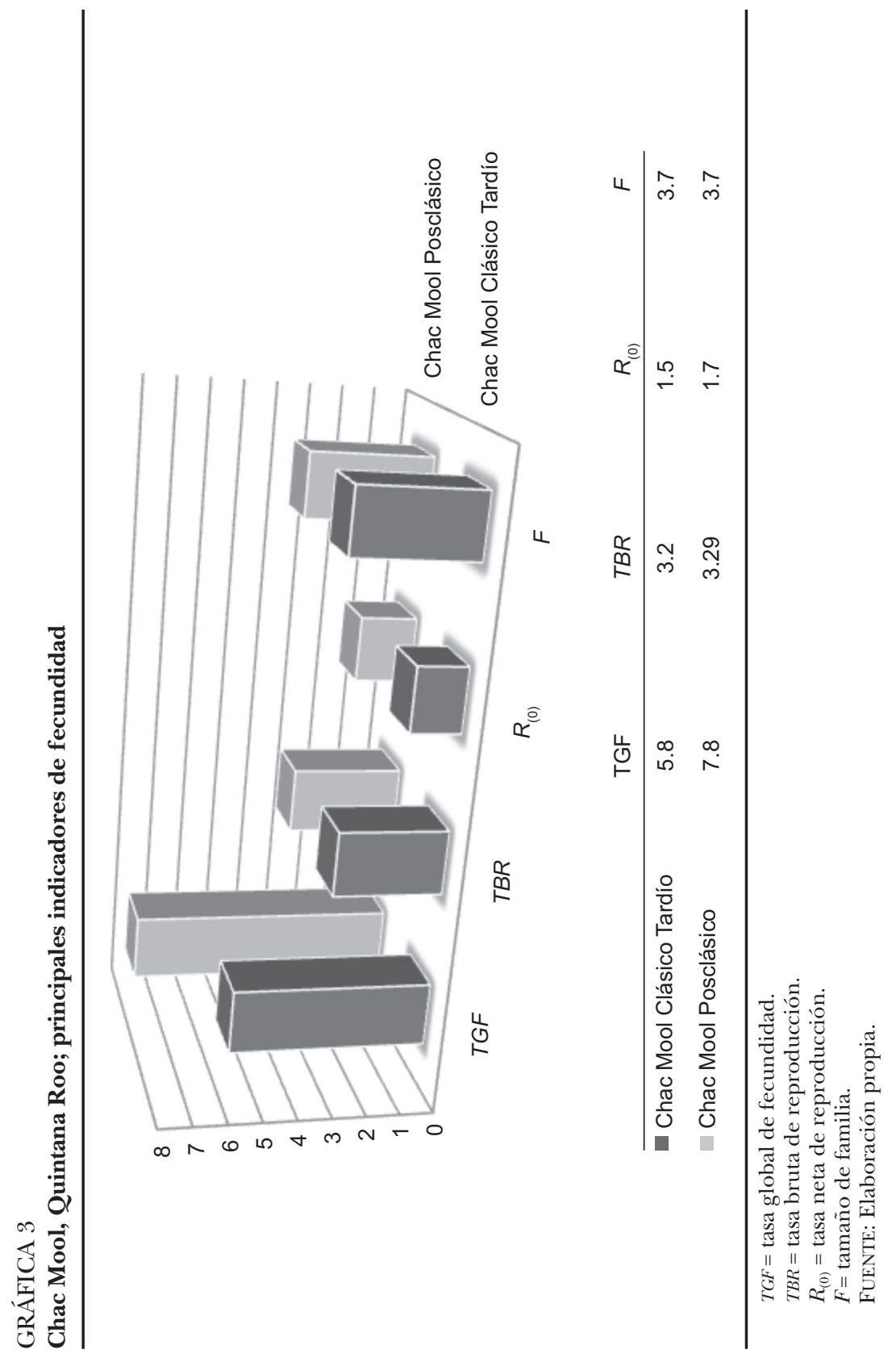


tenido, sin importar si sobrevivieron o no. Esta pregunta se incluyó en los censos mexicanos a partir de 1980, de ahí que podamos usar el método ideado por Louis Henry (1953) para estudiar el comportamiento de la fecundidad marital. Leguina (1992: 225-226) retomó el concepto de Henry y lo adecuó para emplear información censal. Al aplicarse a una cohorte de mujeres que ha completado su familia, es decir, hacia el final de su periodo reproductivo (45 a 49 años), es posible obtener información fidedigna acerca del tamaño final de las mismas, lo que se conoce como descendencia final.

El procedimiento propuesto por Leguina (1992) establece que de una cohorte de $H$ mujeres de un rango de edad: $H_{0}$ no han tenido hijos, $H_{1}$ han tenido $1, \ldots H_{r}$ han tenido $r$. Sean $m_{0}, m_{1}, m_{\mathrm{r}}$ las proporciones de las que han tenido $0,1, \ldots r$ hijos y $m_{1+,} m_{2+} \ldots m_{\mathrm{r}+}$ las proporciones de las que han tenido 1 o más, 2 o más, ... ro más, es decir:

$$
\begin{gathered}
m_{0}=\frac{H_{0}}{H} \quad m_{1+}=\frac{H-H_{0}}{H}=1-m_{0} \\
m_{1}=\frac{H_{1}}{H} \quad m_{2+}=\frac{H-\left(H_{0}+H_{1}\right)}{H}=1-\left(m_{0}+m_{1}\right) \\
m_{r}=\frac{H_{r}}{H} \quad m_{r+}=\frac{H-\left(H_{0}+\cdots+H_{r-1}\right)}{H}=1-\left(m_{0}+m_{1}+\cdots+m_{r+1}\right)
\end{gathered}
$$

La probabilidad de que una mujer de la cohorte haya tenido al menos un hijo será:

$$
\alpha_{0}=m_{1+}
$$

La probabilidad de que una mujer que ha tenido su primer hijo tenga al menos otro es:

$$
\alpha_{1}=\frac{m_{2+}}{m_{1+}}
$$

Así sucesivamente:

$$
\alpha_{r}=\frac{m_{r+1+}}{m_{r+}}
$$

La serie $\alpha_{0}, \alpha_{1} \ldots \alpha_{r}$ recibe el nombre de "probabilidades de agrandamiento de la familia"; mediante su cálculo es posible conocer las etapas de constitución de la familia, "abstracción hecha del tiempo 
necesario para dicha constitución” (Leguina 1992: 226). En otras palabras, estas probabilidades representan la intensidad de los fenómenos $1^{\circ}, 2^{\circ}, \ldots$ résimo hijo. Así $A_{0}$ representa la proporción de mujeres que han tenido al menos un hijo, $\alpha_{0} \alpha_{1}$ será la proporción de las que han tenido al menos dos, $\alpha_{0} \alpha_{1} \alpha_{2}$ la proporción de las que han tenido al menos tres. Este cálculo es lo que Leguina considera como tasas de fecundidad por orden de nacimiento. La suma de dichas tasas representa la descendencia final efectiva $(D \omega)$ de la cohorte de mujeres en cuestión.

Para este trabajo seleccionamos la información sobre fecundidad registrada en los censos de población de 1980, 1990 y 2000 para el estado de Quintana Roo. En específico la información sobre el número de hijos reportados por las mujeres del grupo de edad 45-49. El análisis se hizo en dos ámbitos, el estatal y el municipal.

\section{Resultados}

Las probabilidades de agrandamiento de la familia calculadas para tres generaciones de mujeres, aquellas nacidas entre 1930 y 1935 (que tenían entre 45 y 49 años en 1980); las nacidas entre 1940 y 1945 (45 a 49 años en el censo de 1990) y las que nacieron entre 1950 y 1955 ( 45 a 49 años en el censo de 2000), se presentan en el cuadro 2. Los cálculos se hicieron en el ámbito estatal, considerando todos los municipios, tanto urbanos como rurales (INEGI, 1980, 1990 y 2000).

De las mujeres censadas en 1980, entre la edad de 45 y 49 años $\left(\alpha_{0}\right)$ que declararon haber tenido alguna vez hijos, $96.8 \%$ tuvo al menos un hijo; en 1990 la cifra descendió a $95.7 \%$ y en 2000 a 95\%, lo que implica que el porcentaje de mujeres sin hijos aumentó de 4 a $5 \%$ entre 1980 y 2000 respectivamente. De las mujeres con al menos un hijo $\left(\alpha_{1}\right)$ en 1980, $95.75 \%$ tuvo un segundo hijo como mínimo; en 1990 el porcentaje fue de $95.1 \%$ y en el 2000 de $93.8 \%$. Estas cifras indican que no todas las mujeres con un hijo tuvieron el segundo, y la diferencia más evidente se presenta en el año 2000, cuando $6.2 \%$ de las mujeres con un hijo optó por no tener otro. De acuerdo con los resultados del cuadro 2, la probabilidad de tener una familia numerosa era muy alta en 1980, ya que de las mujeres con diez hijos $\left(\alpha_{10}\right) 76.68 \%$ tuvo el undécimo hijo; para la siguiente década las probabilidades se redujeron ya que de las mujeres con ocho hijos $\left(\alpha_{8}\right), 75 \%$ tuvo un noveno, y de las mujeres con 10 hijos sólo $66 \%$ tuvo el undécimo. 
Hernández y Márquez, LOS ESTUDIOS DE FECUNDIDAD

CUADRO 2

Probabilidades de agrandamiento de la familia para tres generaciones de mujeres de Quintana Roo. Resultados estatales

\begin{tabular}{cccc}
\hline Probabilidades & 1980 & 1990 & 2000 \\
\hline$\alpha_{0}$ & 0.9684 & 0.9575 & 0.9506 \\
$\alpha_{1}$ & 0.9575 & 0.9516 & 0.9383 \\
$\alpha_{2}$ & 0.9410 & 0.9137 & 0.8446 \\
$\alpha_{3}$ & 0.9171 & 0.8790 & 0.7627 \\
$\alpha_{4}$ & 0.8890 & 0.8433 & 0.7400 \\
$\alpha_{5}$ & 0.8860 & 0.8368 & 0.7328 \\
$\alpha_{6}$ & 0.8748 & 0.7899 & 0.7059 \\
$\alpha_{7}$ & 0.8242 & 0.7553 & 0.7107 \\
$\alpha_{8}$ & 0.8037 & 0.7069 & 0.6687 \\
$\alpha_{9}$ & 0.7668 & 0.6667 & 0.6198 \\
$\alpha_{10}$ & 0.7012 & 0.6525 & 0.5946 \\
$\alpha_{11}$ & 0.7215 & 0.6194 & 0.6084 \\
$\alpha_{12}$ & 0.5829 & 0.5337 & 0.5201 \\
\hline
\end{tabular}

FUENTE: Cálculos propios a partir de la información censal del estado de Quintana Roo para los años 1980, 1990 y 2000.

El año 2000 marca diferencias notables en todas las probabilidades, al preferirse las familias menos numerosas: del total de mujeres con cuatro hijos $\left(\alpha_{4}\right) 76 \%$ tuvo un quinto hijo; aun así, la mitad de las mujeres con 13 hijos optó por tener otro, situación que es generalizada en los tres años considerados.

Al graficar las probabilidades de agrandamiento obtenidas en el ámbito estatal, podemos observar que las correspondientes a 1980 y 1990 tienen una forma convexa a partir de la probabilidad de tener el primer hijo hasta la de tener el octavo, lo que significa ausencia de control de nacimientos en este lapso. A partir del noveno hijo la proporción de mujeres que tuvieron un décimo hasta un duodécimo fue menor. La curva que corresponde al año 2000 tiene forma cóncava, indicador del control de nacimientos.

El cuadro 3 muestra los resultados de los cálculos para obtener los niveles de fecundidad por orden de nacimiento, que son también probabilidades derivadas de los cálculos anteriores. En estos resultados todavía son más claros los cambios de las tendencias en cuanto al nú- 

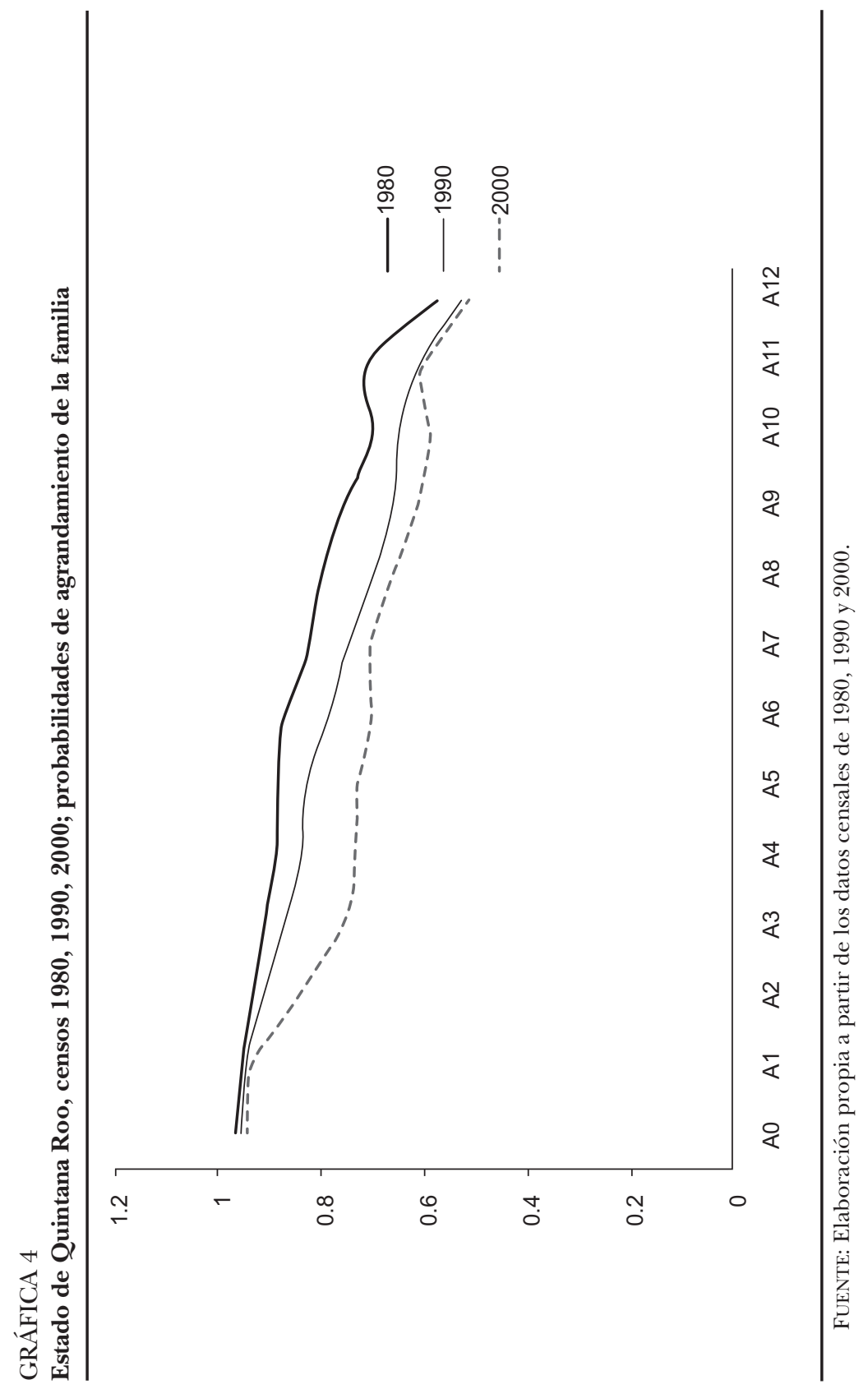
CUADRO 3

Fecundidad por orden de nacimiento y descendencias finales $(D \omega)$ calculada para las mujeres del estado de Quintana Roo; censos 1980,1990 y 2000

\begin{tabular}{clll}
\hline Orden de nacimiento & 1980 & 1990 & 2000 \\
\hline $1^{\mathrm{o}}$ & 0.9684 & 0.9575 & 0.9506 \\
$2^{\mathrm{o}}$ & 0.9273 & 0.9112 & 0.8919 \\
$3^{\mathrm{o}}$ & 0.8726 & 0.8326 & 0.7534 \\
$4^{\mathrm{o}}$ & 0.8003 & 0.7318 & 0.5746 \\
$5^{\mathrm{o}}$ & 0.7114 & 0.6171 & 0.4252 \\
$6^{\mathrm{o}}$ & 0.6303 & 0.5164 & 0.3116 \\
$7^{\circ}$ & 0.5514 & 0.4079 & 0.2199 \\
$8^{\circ}$ & 0.4545 & 0.3081 & 0.1563 \\
$9^{\mathrm{o}}$ & 0.3653 & 0.2178 & 0.1045 \\
$10^{\circ}$ & 0.2801 & 0.1452 & 0.0648 \\
$11^{\mathrm{o}}$ & 0.1964 & 0.0947 & 0.0385 \\
$12^{\mathrm{o}}$ & 0.1417 & 0.0587 & 0.0234 \\
$13^{\circ}$ & 0.0826 & 0.0313 & 0.0122 \\
$D \omega$ & 7.0 & 5.8 & 4.5 \\
\hline
\end{tabular}

FUENTE: Cálculos propios a partir de la información censal del estado de Quintana Roo para los años 1980, 1990 y 2000.

mero de hijos. La suma de todas estas probabilidades es el número promedio de hijos por mujer, que es similar a la tasa global de fecundidad (TGF).

Si seleccionamos las probabilidades cercanas a $50 \%$ como el punto de cambio de la tendencia, observamos que de acuerdo con la información del censo de 1980 las probabilidades de tener el séptimo hijo eran de 55.14\%; en 1990 la probabilidad de tener el sexto hijo era de $51.64 \%$ y para el censo de 2000 había $57 \%$ de probabilidades de tener un cuarto hijo. Si la tendencia era reducir el número de hijos, obviamente la descendencia final efectiva también se redujo de 7.0 hijos en 1980, a 4.5 hijos por mujer en el año 2000.

Las descendencias finales $(D \omega)$ calculadas para los ámbitos estatal y municipal se muestran en el cuadro 4 . En el nivel estatal la descendencia final va, como ya vimos, de 7.0 a 4.5 hijos por mujer, lo que representa niveles moderados de fecundidad de acuerdo con su ubi- 
CUADRO 4

Descendencias finales para mujeres del estado de Quintana Roo y sus municipios; censos 1980-2000

\begin{tabular}{lcccc}
\hline Lugar & 1980 & 1990 & 2000 & Promedio \\
\hline Quintana Roo & 7.0 & 5.8 & 4.5 & 5.8 \\
Cozumel & 6.3 & 5.3 & 3.9 & 5.2 \\
Carrillo Puerto & 8.4 & 6.7 & 6.4 & 7.2 \\
Isla Mujeres & 6.7 & 4.9 & 3.9 & 5.2 \\
Othón Blanco & 6.8 & 6.1 & 4.6 & 5.8 \\
Benito Juárez & 6.6 & 5.2 & 4.0 & 6.2 \\
Morelos & 7.8 & 6.8 & 7.0 & 7.2 \\
Lázaro Cárdenas & 6.8 & 6.3 & 5.8 & 6.3 \\
Solidaridad & - & - & 4.8 &
\end{tabular}

FUENTE: Cálculos propios a partir de la información censal del estado de Quintana Roo para los años 1980, 1990 y 2000.

cación en la etapa intermedia de la transición demográfica mexicana (Juárez y Quilodrán, 1996; Mier y Terán y Rabell, 1993).

$\mathrm{Al}$ efectuar el análisis por municipio es posible observar una tendencia a disminuir el número de hijos. Sin embargo los municipios de Felipe Carrillo y Puerto Morelos mantuvieron descendencias finales numerosas: en treinta años este indicador transitó de 8.2 a 6.4 para el primer municipio y de 7.8 a 7.0 en el segundo, con un promedio de 7.2 hijos por mujer, que al momento de los censos estaban al final de su vida reproductiva y supuestamente habían terminado de formar su familia. Otros dos municipios, el de Lázaro Cárdenas y el de Miguel Hidalgo, también conservaron un elevado número de hijos, 6.2 y 6.3 respectivamente; el resto de los municipios pasó de seis a cuatro hijos en promedio.

\section{Discusión}

Los resultados obtenidos a partir de la información censal contemporánea coinciden con los calculados para el asentamiento prehispánico de Chac Mool, aunque habría que hacer varias precisiones. El método de Henry no supone el impacto de la mortalidad femenina, pues considera a aquellas mujeres que efectivamente sobrevivieron hasta la 
menopausia y que directamente declararon el número de hijos. En el caso de Chac Mool y del modelo empleado, el número de mujeres se simula a partir de una distribución observada. Por otro lado hay que recordar que las series no representan al total de población que habitó en ese asentamiento prehispánico y que hay subrepresentación de individuos para el periodo del Clásico Tardío. Aun así, el efectuar los cálculos con una tasa de crecimiento positiva permite subsanar muchas de estas deficiencias, y también es probable que la fecundidad de estas mujeres haya sido más elevada, tal y como lo demuestran algunos estudios de corte antropológico que se han realizado en la selva lacandona en Chiapas y en la comunidad de Yalcobá en Yucatán (Daltabuilt Godás et al., 1994 y 1997). Tales estudios revelaron que las mujeres mayores de cuarenta años suelen rechazar el uso de anticonceptivos y tienen familias numerosas. Hay familias integradas por siete a nueve hijos en promedio y con altas tasas de mortalidad infantil, de manera que en cada hogar visitado se había perdido al menos un hijo durante sus primeros años de vida. De ser esto así, el cálculo de hijos por mujer se elevaría a casi 10 hijos, lo que coincidiría con el comportamiento reproductivo de una población cuya edad promedio al matrimonio es de 17 años y de 19 al nacimiento del primer hijo (Hernández Espinoza, 2006). En ausencia de control de nacimientos, al término de la vida reproductiva de una mujer su descendencia completa llega en muchos casos a los 12 hijos.

Entre los escasos trabajos de salud y nutrición sobre la población maya contemporánea tenemos los reportes de Berrío Gómez (1995), quien refiere que la mortalidad en los primeros tres años de vida es alta debido a las infecciones respiratorias y gastrointestinales, a los padecimientos virales y las fiebres palúdicas.

Los avances de la medicina han disminuido las probabilidades de muerte de las mujeres en edad reproductiva, pero de acuerdo con los resultados paleodemográficos para Chac Mool, esta probabilidad podría haber sido de 0.60 al principio de la etapa reproductiva, y de 0.50 a los 30 años de edad, lo que probablemente contribuyó a mantener moderados los niveles de fecundidad. La escasa sobrevivencia femenina al proceso de parto constituye uno de los hechos documentados por historiadores de la población como factores del escaso crecimiento demográfico antes de la revolución industrial (Coale y Watkins, 1986; Coale et al., 1983; Knodell y Van del Walle, 1979).

En el caso de las poblaciones indígenas mexicanas la alta mortalidad infantil es todavía un problema de salud pública (González Cer- 
vera y Cárdenas Elizalde, 1992). Si bien las campañas de vacunación han frenado la mortalidad durante la infancia, en menores de un año aún es alta, sobre todo como consecuencia de la desnutrición y de las constantes infecciones parasitarias, principalmente entre las poblaciones que están al margen del desarrollo cuyas condiciones higiénicas son deplorables y reciben una deficiente atención social (Gómez de León Cruces y Partida Bush, 2001: 104-108).

Por estas razones suponemos que entre las poblaciones bajo el régimen demográfico antiguo, como la maya, los niveles de fecundidad eran altos y así se reponía a los hijos muertos durante los primeros años o meses de vida, y compartimos lo que Livi-Bacci (2002: 14) menciona al respecto: "el régimen demográfico antiguo era ineficaz: para que una mujer tuviera cuatro hijos sobrevivientes a los 15 años, debía tener al menos una docena de ellos".

Los altos niveles de fecundidad no implican que las familias fueran cuantiosas por el número de hijos, pues los riesgos de la vida cotidiana hacían que muchos de ellos no sobrevivieran para a su vez reproducirse; eran amplias porque se trataba de familias extendidas. El modelo Weiss propone que, dentro de un esquema de alta fecundidad, alrededor de cuatro hijos rebasaban los 15 años, edad promedio e hipotética de entrada al matrimonio y que variaba de acuerdo con los estilos de vida de cada sociedad.

Este ejercicio, desarrollado a partir de datos censales contemporáneos, permite tener referentes en cuanto al número de hijos que pudieran haber tenido las mujeres en el pasado, habida cuenta de los riesgos que implicaba vivir sin antibióticos y a merced de ambientes físicos y sociales problemáticos, como los causados por la contaminación del agua y de los alimentos. El caso de Chac Mool, un asentamiento maya de Quintana Roo, fue seleccionado para evidenciar la problemática sobre la distribución de los individuos representados en la muestra, sin embargo también deja en claro que el método estadístico que hemos aplicado durante todos estos años para obtener información de diversos grupos prehispánicos mesoamericanos permite tener un acercamiento confiable a los niveles de fecundidad y mortalidad que pudieron haber tenido estas comunidades en el pasado. Los resultados deben ser interpretados considerando el contexto biocultural y físico en el que vivió cada una de estas poblaciones. 
Hernández y Márquez, LOS ESTUDIOS DE FECUNDIDAD

\section{Bibliografía}

Berrío Gómez, Marelvy Isabel (1995), "Fecundidad y proletarización”, tesis de licenciatura en Antropología Física, México, Escuela Nacional de Antropología e Historia.

Brass, William (1975), Methods for Estimating Fertility and Mortality from Limited and Defective Data, Chapel Hill, University of London.

Coale, Ansley J. y Susan C. Watkins (1986), The Decline in Fertility in Europe, Princeton, Princeton University Press.

Coale, Ashley J., Paul Demeny y Bruce Vaughan (1983), Regional Model Life Tables and Stable Populations, Nueva York, Academic Press.

Daltabuilt Godás, Magalí (1992), Mujeres mayas, trabajo, fecundidad y nutrición, México, Universidad Nacional Autónoma de México.

Daltabuilt Godás, Magalí, Marelvy Bérrio y Lucía Garzón (1997), “Conducta reproductiva e ideales de la fecundidad en una comunidad maya de Yucatán”, Estudios de Antropología Biológica, vol. 6, pp. 129-143.

Daltabuilt Godás, Magalí, Luz María Vargas, Enrique Santillán y Héctor Cisneros (1994), "Mujeres de la Selva Lacandona”, en Magalí Daltabuit Godás (coord.), Mujer rural y medio ambiente en la Selva Lacandona, Cuernavaca, Centro Regional de Investigaciones Multidisciplinarias, UNAM, pp. 59-77.

Giannisis, Dimitrios (2004), "Aspectos de la vida cotidiana de una población costera de Quintana Roo, durante el Posclásico. La población de Chac Mool”, tesis de maestría, México, Escuela Nacional de Antropología e Historia.

Gómez de León Cruces, José y Virgilio Partida Bush (2001), "Niveles, tendencias y diferenciales de la mortalidad”, en José Gómez de León Cruces y Cecilia Rabell Romero (coords.), La población de México. Tendencias y perspectivas sociodemográficas hacia el siglo XXI, México, Consejo Nacional de Población / Fondo de Cultura Económica, pp. 81-108.

González Cervera, Alfonso y Rosario Cárdenas Elizalde (1992), La medición de la mortalidad infantil. Los problemas y alternativas, México, Universidad Autónoma Metropolitana, Unidad Xochimilco.

González Licón, Ernesto y Rafael Cobos Palma (2006), "El entorno sociopolítico de Chac Mool, Quintana Roo, durante el Clásico Tardío y el Posclásico”, en Lourdes Márquez Morfín, Patricia Olga Hernández Espinoza y Ernesto González Licón (coords.), La población costera maya de Chac Mool. Perfil biocultural y dinámica demográfica, México, Escuela Nacional de Antropología e Historia, pp. 27-45.

González Licón, Ernesto y Enrique Terrones González (2003), “Género y desigualdad social entre los mayas de Chac-Mol, Quintana Roo durante el Posclásico, a través de sus prácticas funerarias", ponencia presentada en el XII Coloquio Internacional de Antropología Física "Juan Comas", INAH / UNAM / AMAB, La Trinidad, Tlaxcala, 9 a 13 de noviembre.

Henry, Louis (1953), Fecondité des mariages. Nouvelles méthodes de mesure, París, PUF. 
Hernández Espinoza, Patricia Olga (2006), La regulación del crecimiento de la población en el México Prehispánico, México, Instituto Nacional de Antropología e Historia.

Hernández Espinoza, Patricia Olga y Lourdes Márquez Morfín (2003), "La fecundidad como motor del cambio demográfico: el caso de las poblaciones prehispánicas mesoamericanas”, en Sergio Sánchez Díaz y Silvia Araceli Prado Camacho (coords.), Primer Foro de Investigación Científica en la ENAH, México, Escuela Nacional de Antropología e Historia, pp. 239-254.

Hernández Espinoza, Patricia Olga y Lourdes Márquez Morfín (2005), "Las mujeres de Jaina y su fecundidad”, ponencia presentada en el XIII Coloquio Internacional de Antropología Física "Juan Comas", INAH / El Colegio de la Frontera Sur / UNAM / AMAB / Campeche, 6 a 12 de noviembre.

Márquez Morfín, Lourdes y Patricia Olga Hernández Espinoza (2006), “Transición al Posclásico y su efecto en la salud, nutrición y condiciones de vida de algunos pobladores de Chac Mool”, en Lourdes Márquez Morfín, Patricia Olga Hernández Espinoza y Ernesto González Licón (coords.), La población maya costera de Chac Mool. Análisis biocultural y dinámica demográfica en el Clásico Terminal y Posclásico, México, Conaculta / INAH, pp. 113-160.

Hernández Espinoza, Patricia Olga y Lourdes Márquez Morfín (2006a), "Fecundidad y sobrevivencia: perfil paleodemográfico de la población maya prehispánica de Chac Mool, Quintana Roo”, en Lourdes Márquez Morfín, Patricia Olga Hernández Espinoza y Ernesto González Licón (coords.), La población costera maya de Chac Mool: Análisis biocultural y dinámica demográfica en el Clásico Tardío y el Posclásico, México, Conaculta / INAH, pp. 81-112.

Hernández Espinoza, Patricia Olga y Lourdes Márquez Morfín (2006b), "La población maya prehispánica. Una interpretación sobre su dinámica demográfica”, en Lourdes Márquez Morfín y Patricia Olga Hernández Espinoza (coords.), Salud y sociedad en el México prehispánico y colonial, México, Conaculta / INAH, pp. 103-126.

Hernández Espinoza, Patricia Olga y Lourdes Márquez Morfín (2007), "El escenario demográfico de Jaina prehispánica durante el Clásico”, en Patricia Olga Hernández Espinoza y Lourdes Márquez Morfín (coords.), La población prehispánica de Jaina. Estudio osteobiográfico de 106 esqueletos, México, ENAH, INAH, pp. 33-62.

Hill, Kim y A. Magdalena Hurtado (1996), Ache Life History. The Ecology and Demography of a Foraging People, Nueva York, Aldine de Gruyter.

Howell, Nancy (2001), The Demography of the Dobe!Kung, Nueva York, Academic Press.

INEGI (1980), X Censo General de Población y Vivienda, México, Instituto Nacional de Estadística, Geografía e Informática. 
INEGI (1990), XI Censo General de Población y Vivienda, México, Instituto Nacional de Estadística, Geografía e Informática.

INEGI (2000), XII Censo General de Población y Vivienda, México, Instituto Nacional de Estadística, Geografía e Informática.

Juárez, Fátima y Julieta Quilodrán (1996), "Mujeres pioneras del cambio reproductivo en México”, en Fátima Juárez, Julieta Quilodrán y María Eugenia Zavala de Cosío (coords.), Nuevas pautas reproductivas en México, México, El Colegio de México, pp. 97-117.

Knodell, John y Etienne Van der Walle (1979), "Lessons from the Past: Policy Implications of Historical Fertility Studies", en Ansley J. Coale y Susan C. Watkins (coords.), The Decline of Fertility in Europe, Princenton, Princenton University Press, pp. 420-449.

Leguina, Joaquín (1992), Fundamentos de Demografía, Barcelona, Siglo XXI.

Livi-Bacci, Massimo (2002), Historia mínima de la población mundial., Barcelona, Ariel.

Márquez, Lourdes y Patricia Hernández (2001), Principios básicos, teóricos y metodológicos de la Paleodemografía, México, Escuela Nacional de Antropología e Historia.

Márquez Morfín, Lourdes y Patricia Olga Hernández Espinoza (2006), "Transición al Posclásico y su efecto en la salud, nutrición y condiciones de vida de algunos pobladores de Chac Mool”, en Lourdes Márquez Morfín, Patricia Olga Hernández Espinoza y Ernesto González Licón (coords.), La población maya costera de Chac Mool. Análisis biocultural y dinámica demográfica en el Clásico Terminal y Posclásico, México, Conaculta / INAH, pp. 113-160.

McKillop, Heather (2002), Salt. White Gold of the Ancient Maya, Gainesville, The University Press of Florida.

Meindl, Richard S. y Katheryn F. Russell (1998), "Recent Advances in Method and Theory in Paleodemography", Annual Review of Anthropology, vol. 27, pp. 375-399.

Mier y Terán, Martha (1998), "Formación de descendencias y los determinantes próximos de la fecundidad. Generaciones femeninas 1927-1936 en dos regiones de México", en Héctor Hernández Bringas y Catherine Menkes (coords.), La población de México al final del siglo Xx (V Reunión de Investigación Demográfica en México), Cuernavaca, Somede / CRIM, UNAM.

Mier y Terán, Martha y Cecilia Rabell (1993), "Inicio de la transición de la fecundidad en México. Descendencias de mujeres nacidas en la primera mitad del siglo XX", Revista Mexicana de Sociología, vol. 55, núm. 1, pp. 41-81.

Stuart, Gene, S. y George E. Stuart (coords.) (1993), Los reinos perdidos de los mayas, Barcelona, National Geographic.

Terrones González, Enrique (1998), Informe de la tercera temporada de campo en el sitio arqueológico de Chac Mool, Cancún, INAH.

Weiss, Kenneth W. (1973), Demographic Models for Anthropology, Washington, Society for American Archaeology. 


\section{Acerca de las autoras}

Patricia Olga Hernández Espinoza es licenciada en Antropología Física por la Escuela Nacional de Antropología e Historia (ENAH); maestra en Demografía por El Colegio de México y doctora en Antropología por la ENAH.

Su campo de investigación se centra en el estudio de las poblaciones antiguas en los temas de salud y demografía. Actualmente es investigadora del Centro INAH Sonora, en Hermosillo, donde desarrolla la línea de investigación "Bioarqueología y antropología demográfica". Entre sus publicaciones recientes destacan: "Sintió, se dolió y se murió’. Análisis de la mortalidad en Actopan, Hidalgo (1820-1829)", en América Molina del Villar, Lourdes Márquez Morfín y Claudia Pardo Hernández (coords.), El miedo a morir. Historia de las epidemias, pandemias y endemias de México: análisis de larga duración, México, CIESAS / Instituto Mora / BUAP, 2013; Tamtoc. Esbozo de una antigua sociedad urbana, libro coordinado conjuntamente con Guillermo Córdova Tello y Estela Martínez Mora, México, INAH, 2012; "La antropología demográfica o el estudio antropológico de los hechos vitales de la población”, en Anabella Barragán Solís y Lauro González Quintero (coords.), La complejidad de la antropología física, México, ENAH / Conaculta.

Lourdes Márquez Morfín es licenciada en Antropología Física por la Escuela Nacional de Antropología e Historia (ENAH) y doctora en Historia por el Colegio de México. Fue titular de la Dirección de Antropología Física del INAH (1993-1995), profesora invitada en la Universidad de Pittsburgh (1986) y subdirectora de Investigación en la ENAH (1997-1999). Es especialista en estudios sobre salud, sociedad y paleodemografía en Mesoamérica. Actualmente es titular de la línea de investigación "Bioarqueología y antropología forense" del posgrado de Antropología Física de la ENAH (1996-2014) y forma parte de grupos de investigación sobre epidemias, paleodieta y paleogenética. Es miembro del Sistema Nacional de Investigadores, nivel III.

Ha publicado decenas de artículos y es autora del libro La desigualdad ante la muerte en la Ciudad de México. El tifo y el cólera, México, Siglo XXI, 1994. Coordinó el libro Los niños, actores sociales ignorados. Levantando el velo, una mirada al pasado, México, INAH, 2010. Participó en la coordinación de los siguientes libros: Perfiles demográficos de poblaciones antiguas, con José Gómez de León, México, INAH / Conapo, 1998; Salud y sociedad en el México prehispánico y colonial, con Patricia Hernandez, 
México, ENAH, INAH / Conaculta, 2006; Colecciones esqueléticas humanas en México, con Allan Ortega, México, INAH, 2012. Su trabajo más reciente es El miedo a morir. Endemias, epidemias y pandemias en México: análisis de larga duración, que coordinó con América Molina del Villar y Claudia Pardo Hernández, México, CIESAS / Instituto Mora / BUAP, 2013. 
